\title{
Estudio sobre las organizaciones inteligentes en Andalucía
}

\author{
Study on intelligent organizations in Andalusia
}

Recibido: 31/12/2015

Fátima Romera Hiniesta

Universidad de Sevilla

Aceptado: 19/05/2016

\begin{abstract}
The present paper includes a study on Intelligent Organizations in Andalusia. In this study we analysed the changes that are occurring today and that have their impact on companies and their workers, but we understanding them in relation to organizational learning. We also analyse important elements in the development of the organization, such as management, training and innovation; discovering if the economic crisis that have passed the Andalu sian companies and society in general, affects organizations and identifying the factors that have helped overcome it. We presented distinguishing three blocks of content: First, the theoretical framework, in which we contextualize research in Information Society and Knowledge for, from there, analysing change, focused primarily on the company and the consequent need for innovation, and how it training is essential to $m$ ake these changes in learning, and we know as organizational learning. Second, the empirical study that ocaupies the central part of the study is presented. It covers the entire research process: object of study, process followed, sample, methodology, instrument used, data analysis and results Finally, condusions are presented, trying to provide data to enrich the training within companies through management models based on organizational learning and knowledge management, and in turn leaving open lines of future research in this area.
\end{abstract}

KEY WORDS: Organizations, Knowledge, Enterprises, Learning, Cultural change, Training.

\section{RESUMEN}

El trabajo que presentamos abarca un estudio sobre las Organizaciones Inteligentes en Andaluáa. En este es tu dio analizamos los cambios que se están produciendo en la actualidad y que tienen su repercusión en las empresas y sus trabajadores, pero los analizaremos entendiéndolos en relación con el aprendizaje de la organización. También analizamos elementos importantes en el desarrollo de la organización, tales como la gestión, la formación y la innovación; descubriendo si la crisis económica por la que han pasado las empresas andaluzas y la sociedad en general, repercute en las organizadiones e identificando los factores que las han ayudado a superarla. Lo presentamos distinguiendo tres bloques de contenido: En primer lugar, el marco teórico, en el que contextualizamos la investigación en la Sociedad de la Información y el Conocimiento para, a partir de ahí, analizar el cambio, centrado sobre todo en la empresa y la consecuente necesidad de innovación, y cómo ésta hace imprescindible una formación que convierta estos cambios en aprendizaje, y que conocemos como aprendizaje organizativo. En segundo lugar, se presenta el estudio empírico que ocupa la parte central del estudio. Abarca to do el proceso de investigación: objeto de estudio, proceso seguido, muestra, metodología, instrumento utilizado, análisis de los datos y resultados obtenidos. Finalmente, se exponen las condusiones, intentando aportar datos que enriquezcan la formación dentro de las empresas a través de modelos de gestión basados en el aprendizaje organizativo y la gestión del conocimiento, y a su vez dejando abiertas líneas de investigación futuras en este ámbito.

PALABRAS CLAVE: Organización, Conocimiento, Empresa, Aprendizaje, Cambio Cultural, Formación.

Dirección de correspondencia:

Fátima Romera Hiniesta, Departamento de Didáctica y Organización Educativa, Universidad de Sevilla. e-mail: fatimaromera@yahoo.es 


\section{Introducción}

Mejorar el capital humano impulsa la actividad innovadora en las empresas, lo que se traduce en aumento de la productividad y la competitividad, el círculo se cierra con la influencia (también positiva) de la competitividad en el capital humano.

Es aquí donde consideramos que la formación juega un importante papel como mediadora y facilitadora del cambio. No hablamos de cambios superficiales, nos referimos a transformaciones desde la base, modificar conciencias y esquemas ya establecidos para comenzar a trabajar con otros totalmente nuevos. Consideramos que sólo a través de la formación es la única manera de conseguir este cambio cultural que pretendemos.

Nuestra sociedad está expuesta a grandes cambios, que están transformando la forma de vida de nuestra sociedad desde los cimientos. La incorporación de la mujer al mercado de trabajo, la introducción de las Nuevas Tecnologías, la ilimitada cantidad de información a nuestro alcance, la necesidad de actualización continua, la movilidad laboral, los nuevos entornos laborales, la flexibilidad espacial y temporal, hacen que trabajar no sea hoy lo que era hace unos años. El cambio es una variable presente en nuestra vida laboral y hay que estar preparado para ello, debemos aprender de él.

Los/as profesionales de la formación, tienen mucho que hacer en estos momentos de cambio, en los que la información y el conocimiento son los motores que accionan todos los mecanismos, y donde la adquisición y el desarrollo de competencias, son el eje principal de la adaptación y los que posibilitan el correcto funcionamiento.

\section{Sociedad de la información y conocimiento}

Cuando hablamos de Sociedad de la Información y el Conocimiento, nos referimos a uno de los cambios más relevantes desde la Revolución Industrial, a un nuevo modelo de desarrollo tecnológico, económico y social que ha impactado -y continúa haciéndolo crecientemente- en la vida de los individuo. Este cambio exige a las personas nuevas competencias que les ayuden a desenvolverse en esta nueva realidad.

Todos estos cambios que la Sociedad de la Información y el Conocimiento han supuesto, han significado también, una nueva forma de ver la empresa y su gestión y han visto la luz nuevos modelos organizativos, basados en la gestión del conocimiento y la ética en las organizaciones. Se persigue un ideal: las organizaciones inteligentes, donde lo primordial sean las personas, el capital humano, y su capacidad de aprender e innovar.

La formación en la empresa, el aprendizaje permanente y situado en la organización, son los elementos básicos del cambio cultural.

Por tanto, las empresas demandan, aún sin saberlo, esta formación, este aprendizaje organizativo. Se considera que una organización aprende cuando promueve el aprendizaje de sus miembros y lo aprovecha para mejorar e innovar permanentemente; es decir, cuando concibe el aprendizaje como un medio para adaptarse a los procesos de cambio.

En resumen, innovación y cambio son dos conceptos que van de la mano, y que definen una de las características de la cultura y sociedad actual Debemos ver las crisis como contextos en los que florece la innovación, aunque sea doloroso. Si anteriormente no ha habido innovación es porque no ha habido necesidad de ella. No tenemos asumida la importancia de la innovación, ya que no da frutos de un día para otro. Es una inversión a largo plazo y difícil de mantener

\section{Modelos de organización}

Podemos afirmar que en la actualidad conviven distintos modelos organizativos en función de diversos factores. Siguiendo la clasificación que propone Mintzberg podemos diferenciar entre: 
a) La estructura simple, es eso, simple, informal, flexible. Son empresas pequeñas y medianas en que las actividades giran alrededor del director general que realiza la supervisión directa de los empleados. (Ej. Tienda de zapatos)

b) La burocracia maquinal, en este tipo de organizaciones, se da una proliferación de reglas, normas y comunicación formal. El poder de decisión está centralizado en el ápice estratégico y posee una elaborada estructura administrativa. (Ej. Oficina de correo)

c) La burocracia profesional. Todas cuentan con las habilidades y los conocimientos de profesionales operativos para funcionar y todas producen bienes y servicios normalizados. (Ej. Universidad)

d) En el caso de la organización divisional o diversificada se presenta una estructura basada en divisiones autónomas, cada de ellas con una estructura propia, débilmente acopladas bajo una dirección administrativa única. (Ej. Multinacionales)

e) La adhocracia significa cualquier estructura altamente flexible capaz de adaptarse continuamente a las condiciones cambiantes del ambiente. Es una organización fluida, descentralizada, de equipos multidisciplinarios coordinados mediante adaptación mutua, con directivos integradores y estructura matricial. En este tipo de organización se fomenta la innovación por lo que suele presentarse en el mundo de la alta tecnología. (Ej. Productora cinematográfica)

\section{Objeto de estudio}

El objeto de estudio, que nos ha guiado a lo largo de toda la investigación, abarca, en primer lugar, "conocer los factores que influyen en los cambios producidos en la empresa andaluza tras su incorporación a la Sociedad de la Información y el Conocimiento". Esto supone un primer nivel, superficial, macro, que nos ha ayudado a configurar un esquema amplio y general. Y además, nos ha servido como punto de partida que nos permite profundizar hasta un nivel más concreto y específico para "detectar tipologías de empresas en función de estos factores".

Ambos objetivos nos conducen a un tercero y concluyente, que ha supuesto "crear una herramienta autodiagnóstica para las empresas que les permita conocer a qué tipología de empresa pertenecen $y$ las oriente bacia un itinerario formativo determinado".

Para abordar estos objetivos generales, se plantearon una serie de objetivos específicos. Los expondremos en relación con cada uno de ellos:

a) Conocer los factores que influyen en los cambios producidos en la empresa andaluza tras su incorporación a la Sociedad de la Información y el Conocimiento.

1. Descubrir qué ha supuesto la incorporación del ordenador e Internet a las empresas andaluzas.

2. Identificar los componentes de la cultura de las empresas: innovación, gestión de Recursos Humanos, clima laboral, formación, TIC, responsabilidad social corporativa; considerados elementos claves de las empresas que propician o pueden llegar a propiciar el cambio cultural.

3. Identificar qué factores son más valorados en las organizaciones.

4. Analizar si existe un cambio cultural en la empresa propiciado por las TIC e Internet.

5. Descubrir qué papel juega la formación en la empresa andaluza.

6. Conocer qué medidas o factores se consideran fundamentales para superar la actual situación de crisis económica.

b) Detectar tipologías de empresas en función de estos factores.

1. Obtener el perfil medio de las empresas andaluzas.

2. Analizar los conglomerados de empresas resultantes en función de los factores principales del estudio (gestión, formación, cambio cultural, innovación y crisis económica). 
3. Representar gráficamente estas agrupaciones de empresas.

c) Crear una herramienta autodiagnóstica para las empresas que les permita conocer a qué tipología de empresa pertenecen y las oriente hacia un itinerario formativo determinado.

1. Dotar a las empresas de una herramienta simple, de fácil aplicación, que les permite conocer su posición dentro de las tipologías del empresariado andaluz y que les conceda una cierta autonomía para optar por un itinerario formativo.

2. Desarrollar un itinerario formativo general, con pautas sencillas que permita a las empresas realizar sus propios planes de formación adaptados a sus necesidades pero conociendo en qué niveles de profundidad deben situarse en cada uno de los factores estudiados.

3. Concienciar de la importancia de estructurar y planificar la formación, de acuerdo a las pautas que marquen el mercado, la sociedad y las necesidades de los propios implicados en el proceso formativo.

Estos objetivos van a lograrse a través del estudio de:

a) El cambio cultural propiciado por la introducción de nuevos componentes, tales como las TIC, Internet y la incorporación de la mujer

b) El papel de la formación como agente mediador y facilitador del cambio.

c) La gestión empresarial

d) Los factores de crisis económica

e) La distinta percepción que tienen las empresas de los factores estudiados en función de diferentes rasgos intrínsecos de las mismas y de sus miembros (edad, sexo, cluster de pertenencia...).

f) El poder discriminante que tienen los factores analizados

Además de la comprobación de estos planteamientos de partida, también estudiaremos los componentes de la cultura, que hemos considerado indicadores del cambio. Valorando la importancia que la empresa da a cada uno. Con todo ello obtendremos las diferentes tipologías de empresas que en la actualidad se dibujan en nuestra comunidad autónoma y propondremos los distintos itinerarios formativos que deberán marcarse para cada una de ellas.

\section{Proceso de investigación}

De esta forma el proceso seguido ha sido el siguiente:

a) Partiendo de la contextualización de la realidad, a través del análisis teórico de los temas,

b) Optamos por una metodología de carácter descriptivo / analítico para nuestro estudio empírico.

c) Para lo que empleamos como instrumento de recogida de datos, un cuestionario realizado ad hoc para las empresas, ya que pretendíamos estudiarlas en su contexto sin someterlas a manipulación alguna. El cuestionario está formado por 130 ítems agrupados en 8 dimensiones: Datos personales, datos profesionales, el empleo de las TIC en la empresa, gestión, formación, innovación, factores de cambio cultural en la empresa y factores de crisis económica y su repercusión en la empresa.

\subsection{Muestra}

En este estudio se pretendía trabajar con toda la población. Finalmente el número de cuestionarios recibidos, aunque no muy alto proporcionalmente, nos pareció relevante para poder trabajar. Además con este número de cuestionarios pensábamos que se podían cubrir los objetivos de la investigación. No pretendemos generalizar estos resultados a todo el conjunto de la población, 
puesto que el número de empresas con las que hemos trabajado no nos permite hacer una generalización de todos los resultados.

El proceso seguido fue el siguiente: Se decidió contactar por correo electrónico con las empresas. Tras un arduo trabajo de obtención de los correos electrónicos de contacto de la muestra, obtuvimos un listado que nos permitió contactar con dichas empresas y hacerles llegar el cuestionario del estudio. Así pues se decidió pasar al envío de los cuestionarios, acompañados de una carta explicativa del estudio que estábamos llevando a cabo y la importancia de su colaboración. Los cuestionarios fueron enviados por correo electrónico, ya que su formato digital hace más cómoda y rápida su cumplimentación. Este proceso fue lento y requiso un seguimiento continuado de los cuestionarios, recordando la importancia que para nosotros tenía que colaborasen y nos devolviesen el cuestionario completado en un tiempo prudencial. Finalmente, logramos recuperar un total de 140 cuestionarios, de los que sólo 125 eran válidos y son los que componen la población real del estudio:

- Nos encontramos con una edad adulta media, con una media predominante de edad entre (30 - 50 años).

- Aproximadamente tres cuartas partes de las personas que han contestado el cuestionario son hombres.

- Es un dato significativo para nuestra investigación el comprobar cómo la formación es un valor reconocido en las empresas, al menos para acceder a los puestos de responsabilidad, ya que más del $70 \%$ de la muestra recogida posee estudios universitarios.

- Las carreras técnicas encabezan con gran diferencia (60\%8\%) la tipología de estudios de los encuestados, seguidos a gran distancia de las Ciencias Sociales (20’8\%). Poco representativos son los porcentajes obtenidos para el resto de ramas o especialidades.

- Más de un tercio (36\%), posee estudios de posgrado, lo que nos indica el nivel de cualificación existente en nuestras empresas y el valor que se le da a la misma. La formación continua del resto se apoya en la empresa, tanto en la formación que se ofrece dentro de la misma (36'8\%), como el aprendizaje que le posibilita la propia experiencia y los conocimientos de los compañeros (18'4\%).

- En nuestra empresa, aún es escaso el porcentaje de trabajadores que ha adquirido una formación continua en el extranjero (4'8\%).

- En nuestra muestra podemos observar cómo aproximadamente la mitad de los encuestados comparte a parte iguales la responsabilidad familiar.

- El nacimiento de los hijos en muchas ocasiones se convierte en un factor determinante, sobre todo para las mujeres, en lo relacionado con su carrera profesional.

- A veces se relaciona con el abandono del trabajo o la retirada temporal del mundo laboral, reducción de jornada, menor disponibilidad y movilidad geográfica.

- Un alto porcentaje de encuestados aún no tiene hijos (27’2\%), aunque lo más habitual en nuestra muestra parecen ser las familias con dos hijos, representando este dato más del $40 \%$ de la misma.

- Existe una amplia representación de puestos, que nos posibilita una visión desde múltiples perspectivas y además en su mayoría con una amplia experiencia profesional.

\subsection{Metodología}

Como punto de partida de la investigación se ha realizado un análisis teórico de los temas objetos de estudio, así como de la situación actual, analizando opiniones de expertos y casos y situaciones concretas que nos ha ayudado a contextualizarnos en la realidad a estudiar. 
Para el estudio empírico se ha aplicado una metodología de carácter descriptivo/analítico, y como instrumento de referencia hemos empleado un cuestionario que nos ha ayudado a obtener información relevante de la muestra de empresas andaluzas.

Nuestro objeto de estudio, las empresas andaluzas, han sido analizadas y estudiadas en su entorno, sin someterlas a intervención o manipulación alguna.

Hemos empleado un cuestionario ad hoc, diseñado específicamente para el estudio y en versión on line que nos ha permitido recabar la información de un modo ágil y ha posibilitado a las empresas cumplimentarlo de un modo cómodo y rápido.

El resultado final ha sido un cuestionario que se compone de 130 ítems agrupados en 8 dimensiones: Datos personales, datos profesionales, el empleo de las TIC en la empresa, gestión, formación, innovación, factores de cambio cultural en la empresa, factores de crisis económica y su repercusión en la empresa.

Cuando analizamos la validez de nuestro constructo a través de una reducción de datos con análisis factorial explicando el método de componentes principales con una rotación varimax cada una de nuestras escalas obtiene una alta varianza a través del menor número posible de componentes.

En cuanto a la evaluación de la fiabilidad de nuestras escalas, nos desprende unos resultados muy positivos, obteniendo que tanto la escala de Gestión, como la de Formación e Innovación tienen una coeficiente Alpha superior a 0.9 por lo que su calificación es excelente.

Las escalas de cambio cultural y factores de crisis económica, obtienen un resultado por encima de 0.8 , lo que calificaría a ambas escalas como buenas.

\section{Análisis de datos}

Recogidos todos los datos nos dispusimos a pasar a su análisis a través del programa estadístico SPSS. Los datos los pasamos a una matriz de respuestas que analizaba 160 variables. Los datos fueron sometidos a diferentes análisis estadísticos en función del objetivo al que pretendían dar respuesta.

Pasaremos a describir detalladamente el plan de trabajo metodológico desarrollado para tal fin:

\section{Objetivo 1:}

Para la consecución de este objetivo, los análisis llevados a cabo a los datos obtenidos en el estudio han sido, en primer lugar, la realización de estadísticos descriptivos que nos permiten describir y representar gráficamente, a través de porcentajes, la realidad estudiada.

Posteriormente, se han aplicado tablas de contingencias, que nos han permitido analizar algunos datos en función de otros, para así poder realizar contrastes que nos han posibilitado conocer la influencia en los resultados de aspectos tales como la edad o el sexo de la persona encuestada.

La prueba de chi-cuadrado de Pearson, también empleada en el estudio, analiza la relación entre dos variables categóricas. Esto indicará si existe una diferencia estadísticamente significativa entre los dos grupos. La importancia indica la probabilidad de que la diferencia se debe a la variable predictora y no al azar.

\section{Objetivo 2:}

Para la consecución de este segundo objetivo, las pruebas empleadas han sido diferentes pero complementarias a las anteriores, lo que nos ha permitido pasar de un nivel de análisis macro, general y descriptivo, a un nivel de mayor profundización, centrado en aspectos más concretos y basado más en el contraste, la factorización...es decir más en la clasificación que en la descripción.

Enumeramos las pruebas realizadas: ANOVA, Kruskal- Wallis, U de Mann Whitney, Análisis Factorial, Conglomera de K medias, Discriminante.

\section{Objetivo 3:}


Para este objetivo nos hemos basado en los resultados extraídos de algunas de las pruebas, para la realización de la herramienta autodiagnóstica, que es un cuestionario Excel que automáticamente calcula la media de las puntuaciones concedidas a cada ítem dentro de un factor, obteniendo así un valor medio, que también de forma automática se pasa a un gráfico en el que aparecen ya representados previamente los tres cluster o conglomerados obtenidos en el estudio. Esto nos permitirá la ubicación de nuestra empresa en uno u otro conglomerado y, por tanto, la recomendación de optar por uno u otro itinerario formativo de los recomendados.

\section{Resultados}

Todas estas pruebas, han dado lugar a una serie de resultados, que pasaremos a exponer de formar organizada en función de los distintos factores:

\subsection{Incorporación de las nuevas tecnologías}

Respecto a la incorporación de las nuevas tecnologías el análisis realizado de la empresa andaluza nos indica que éstas están prácticamente extendidas por todas ellas, siendo habitual la existencia de un ordenador por cada puesto de trabajo y estando generalizado el uso de Internet para contactar con clientes y proveedores.

El uso de las TIC ha facilitado la flexibilidad de tiempos y espacios en el trabajo, provocando el uso de internet cambios tanto en la cultura como en la productividad de la empresa.

Son los mismos trabajadores los que lo reconocen, más del $70 \%$ afirman que hay un cambio en la cultura de la empresa por el uso de Internet.

Es significativo cómo a mayor edad, mayor es la percepción de que ha tenido lugar un cambio en la empresa por el uso de Internet.

Más del $80 \%$ de los encuestados lo corrobora que el uso de las TIC en la empresa facilita la flexibilidad de tiempos y espacios en el trabajo.

Internet también ha supuesto cambios en la productividad y así lo confirman el $75 \% 2 \%$.

Nuevamente la edad juega un papel significativo e interesante. Los datos nos ponen de manifiesto como a mayor edad del trabajador más reconocen los cambios que han tenido lugar en la productividad de la empresa, ya que ellos los han ido viviendo.

Usos concretos de Internet:

a) Para los negocios: no existe excesivo temor a la inseguridad informática y que eso no limita el uso de Internet para los negocios, al menos para el 68\% de la muestra.

b) Para contactar con proveedores y clientes: es un uso con el que están de acuerdo la mayoría (88\% de la muestra).

c) Para el e-comercio: Comprar y vender sin movernos de nuestro sitio: podemos observar cómo es algo que poco a poco se va extendiendo en las empresas. El $46^{\prime} 4 \%$ afirma usarlo para comprar y vender.

d) Para la colaboración entre empresas con negocios comunes: el uso de Internet para la colaboración entre empresas con negocios comunes está cada vez más extendido.

Cuando les preguntamos por otros usos de las tecnologías, encontramos:

a) Redes internas: la Intranet. Observando los resultados, concluimos que entre sus usos los más importantes son la comunicación entre servicios $(52 \% 8 \%$ y la facilitación de la participación del personal en la gestión de la empresa (48’8\%). 
b) Nuevos elementos, nuevas posibilidades: utilidades del Blog. El Blog no es una herramienta tan generalizada, más de la mitad de las empresas aún no disponen de ella (52’8\%). Los que sí lo han incorporado ya, lo usan mayoritariamente para publicidad $\left(19^{\prime} 2 \%\right)$ y para la comunicación con los clientes (17’6\%).

c) ¿Nos sentimos dominadores o dominados por los programas informáticos? Aproximadamente la mitad de la muestra (42’4\%) afirma dominar los programas informáticos.

Llegados a este punto, podemos decir que afirmamos la primera hipótesis que nos planteamos de partida: " $\mathrm{Si}$ en la cultura empresarial introducimos nuevos componentes, que conllevan en sí mismos el concepto de innovación y cambio, éstos deben provocar un cambio cultural en las empresas".

Cuando analizamos los factores de cambio cultural en la empresa, vemos como existen una serie de afirmaciones que alcanzan un alto grado de acuerdo en la gran mayoría de las empresas. A continuación los exponemos por orden de mayor acuerdo:

- La empresa tiene medidas equiparadoras de los dos sexos para el acceso y promoción en el trabajo.

- La directiva/o tiene capacidad de trabajo en equipo.

- La directiva/o transmite la información dentro de la organización y se preocupa de que circule convenientemente por unidades y personas.

- La reducción de la jornada laboral para cuidar a hijos menores de 6 años no influye en las oportunidades de promoción en la empresa.

Las posibilidades de conciliación, son unas de las características más significativas del cambio cultural:

Hemos podido observar cómo las más comunes en la mayoría de empresas son el horario flexible $(44 \%)$ y el teléfono móvil $\left(33^{\prime} 6 \%\right)$. Lo siguen de cerca las posibilidades que les ofrece el contar con ordenador portátil (27’2\%), el permiso de paternidad $\left(25^{\prime} 6 \%\right)$ y la jornada intensiva los viernes $(24 \% \%)$. Las medidas menos frecuentes son la maternidad y periodo de lactancia ampliados $\left(17^{\prime} 6 \%\right)$ y el ADSL en casa $\left(10^{\prime} 4 \%\right)$.

\subsection{Formación}

Otro importante factor estudiado, ha sido la formación. En la actualidad en las empresas andaluzas consultadas, la existencia de un departamento de formación propio, sólo se da en algo menos de un tercio de las mismas $\left(28^{\prime} 4 \%\right)$. Aunque no es muy abundante la presencia de departamentos de formación en las empresas, sí es cierto, que los existentes cuentan, en la mayoría de los casos, con una amplia experiencia, ya que prácticamente la mitad de los mismos (41\% $\%$ \%) tienen una existencia superior a los 10 años. Estos departamentos dependen en la mayor parte de los casos directamente de la Dirección General (47’8\%) o en su defecto, de Recursos Humanos $\left(377^{\prime} \%\right)$.

Los resultados nos muestran que, aunque está bastante equilibrado, despunta la formación proactiva (56'2\%), es decir aquella que se anticipa y se realiza antes de los cambios previstos, frente a la formación reactiva (43\% $\%$ ), que se entiende como aquella que reacciona ante las exigencias del momento y se realiza después.

Por otra parte cuando hablamos de formación en la empresa, hablamos de una formación para todos, pensada y dirigida hacia todos los empleados de la organización, realizándolas con un total al menos de 30 a 50 horas de formación al año $(82$ '5\%).

Lo más habitual es que no existan muchas dificultades para sensibilizar al personal hacia la formación, pero en la cuarta parte de las empresas, sí encontramos esa dificultad (24'8\%). Cuando esta situación se da, las causas principales, por orden de importancia son: 
a) Tiempo escaso para destinar a formación (muy importante para el 31'8\%),

b) Horario fuera de la jornada laboral (considerado causa de mayor importancia para el $273 \%)$

c) No percepción de la necesidad de formación (lo valoran como la primera causa, el 20’9\%).

Debería ser lo habitual que las empresas organizasen su formación en un plan anual, donde partiendo de una detección de necesidades previas se marcasen objetivos para cubrirlas. Pero el análisis resultante de los datos recogidos, nos muestra que la realidad no es ésta para todas las empresas y a pesar de predominar una formación proactiva, más de la mitad de las empresas $\left(56^{\prime} 6 \%\right)$ afirman no poseer plan de formación.

En las empresas que cuentan con un plan de formación anual para todos los trabajadores, podemos afirmar que éste se basa sobre todo en la competitividad de la empresa $(30 \%)$ y en las necesidades de los trabajadores $(20 \%)$.

También es un dato llamativo que el 35\% de los encuestados desconoce en función de qué se basa el plan de formación continua de su empresa, lo que arroja datos de la falta de conocimiento por parte de los trabajadores y de la falta de transmisión por parte de la empresa de todo lo relativo a la formación.

Hemos considerado de interés conocer dentro de la formación empresarial en Andalucía qué áreas son las que más interesan para el desarrollo profesional de sus trabajadores, obteniendo como resultado del estudio, que con gran diferencia sobre el resto, destacan los conocimientos técnicos $(51 \%)$

Es destacable que más de la mitad de los encuestados nos afirman que no existe diferencias por ninguna modalidad formativa $(56 \%)$.

Esta breve visión panorámica de la forma de organizar los planes de formación anuales de las empresas u organizaciones andaluzas, podemos concluirla con lo que las empresas han considerado los tres pilares básicos a los que orientan su formación:

a) elevar el grado de formación general del personal (52’5\%),

b) desarrollar la polivalencia en el puesto de trabajo (23'2\%),

c) y facilitar los cambios técnicos (19’2\%).

Hemos querido profundizar más en este aspecto y preguntar directamente a las empresas acerca de una serie de afirmaciones que ponen en relación ambos conceptos, formación y cambio cultural, y les hemos pedido que nos las valoren. Estas afirmaciones nos ayudarán a visualizar una perspectiva global real de la situación de nuestras empresas entorno a estos dos conceptos:

Entre todas las afirmaciones, las que reciben un mayor acuerdo en un alto porcentaje de las empresas estudiadas son:

- La formación recibida puede aplicarse en el trabajo diario.

- La formación propicia el desarrollo de nuevas habilidades en los trabajadores.

- La empresa posibilita el desarrollo profesional de sus trabajadores dentro de la compañía.

- La empresa da la oportunidad a sus trabajadores de tomar la iniciativa de su propio desarrollo profesional.

- Se valoran los esfuerzos de los trabajadores por mejorar sus puntos débiles.

- Se valora la formación en la empresa, aprovechando la experiencia y el conocimiento distribuido.

- Se atienden a las propuestas de formación individuales dentro de la organización.

- Valoramos la formación que ofrecen organismos especializados.

- Se realizan esfuerzos por parte de los superiores para identificar sus fortalezas y debilidades de sus subordinados. 
En último lugar, y no por ello menos importante, hablando de cambio y formación es importante no olvidar la evaluación del impacto, medir y valorar el cambio concreto que la formación ha propiciado. Cuando les hemos pedido que nos digan con sus propias palabras por qué es importante la formación continua en su empresa, éstas son las respuestas obtenidas:

- Actualización

- Exigencias del mercado

- Adquisición de habilidades

- Desarrollo personal

- Desarrollo profesional

- Apuesta por el talento

- Mejora productividad

- Competitividad

- Mejora empresarial

- Incremento capacidades

- Mejora calidad

- Mejora servicio

- Incremento de la eficacia y eficiencia

- Mantener vivo el conocimiento

- Alcanzar excelencia

\subsection{Gestión}

Cuando pasamos a analizar otro de los factores de estudio como es la GESTIÓN vemos que la visión que tienen los/as directivo/as sobre el trabajo colaborativo es muy alta. El 78’4\% de ellos afirman que se sienten totalmente identificados/as con la visión de la empresa y un $72 \%$ se define por tener una conciencia colectiva orientada hacia la atención al cliente. El 67’2\% de los/as Directivos/as están de acuerdo con que dentro de su Departamento se comparten los objetivos.

a) La gestión de los Recursos Humanos. En las empresas andaluzas representadas en nuestra muestra podemos afirmar con un amplio porcentaje de apoyo que la gestión de los recursos humanos se basa en una negociación con los individuos (72’8\%).

b) ¿Qué se valora más en nuestras organizaciones? El 59'2\% de las empresas estudiadas afirman estar de acuerdo o absolutamente de acuerdo con que en su empresa se trabaja por objetivos. Para el 52\% de las empresas, los incentivos se relacionan con el logro de objetivos. En el $73^{\prime} 6 \%$ de las empresas se reconocen y se valoran las aptitudes de los trabajadores. Un porcentaje similar $(76 \%$ \%) de las empresas reconocen y valoran las actitudes laborales.

\subsection{Innovación}

Si comenzamos a indagar acerca del factor innovación podemos observar cómo la innovación también requiere formación, actualización de conocimientos. Si intercalamos formación e innovación, y preguntamos qué temas en la formación de directivos relacionadas con la innovación son los que más se promueven en su empresa, los resultados nos indican que destacan por igual la formación en creatividad y liderazgo (30’4\%) seguidos de la gestión del tiempo (23'2\%). La responsabilidad social corporativa $(16 \% \%$ ) y la sociedad de la información (12\%) no forman parte de las temáticas más promovidas. En este apartado hemos encontrado diferencias significativas en función de la edad, como puede observarse en el gráfico.

Incidiendo aún más en la innovación, nuestro análisis desvela cómo sólo el 47’3\% de las empresas andaluzas afirman participar en proyectos de $\mathrm{I}+\mathrm{D}$ y apenas un 35 ' $\%$ \% afirman haber 
establecido convenios de colaboración con universidades para proyectos de $\mathrm{I}+\mathrm{D}$, pero es destacable que en un $73^{\prime} 1 \%$ de ellas están de acuerdo en que se estimula la iniciativa de los trabajadores. Dato muy importante por las consecuencias positivas que de ello puede derivarse, tales como la motivación laboral.

Afirmaciones sobre la innovación en las empresas andaluzas, arrojan a la luz algunos datos muy interesantes. Afirmaciones tales como:

- Su empresa aprovecha de forma sistemática las sugerencias y las quejas de sus clientes para generar mejoras e innovaciones de producto o de proceso.

- Su empresa invierte regularmente en tecnología (maquinaria, bienes de equipo, ordenadores...) para conseguir ventajas competitivas.

- La empresa ha optado por la gestión de la calidad total.

- La estrategia de su empresa tiene en cuenta la innovación y la considera como un factor clave para su éxito.

- La innovación en su empresa contempla además del desarrollo de nuevos productoso servicios, la mejora de los procesos.

- Son las que alcanzan al mayor grado de acuerdo en todas las empresas.

6.5. Factores de la crisis económica y su influencia

Podemos concluir, que se está llevando a cabo una gestión empresarial en la empresa andaluza orientada al cambio y que esto está cambiando la forma de gestionar los recursos humanos, la forma de concebir la innovación y la valoración de ciertos "nuevos elementos" dentro de la empresa (iniciativa, colaboración, capacidades, actitudes, formación...), y todo ello está revirtiendo en un buen clima empresarial.

Hemos considerado interesante para nuestro estudio contextualizarlo en el momento económico y social que estamos viviendo. Es por eso, que es de especial interés conocer los factores de crisis y la influencia que están teniendo en la empresa. Muchas empresas reconocen que se han producido cambios en la empresa como consecuencia de la crisis, y uno de ellos ha sido la reducción de plantilla $\left(56^{\prime} 5 \%\right)$. Es muy significativo destacar como un altísimo porcentaje de empresas indican que han sido la innovación y la formación algunas de las medidas tomadas para adaptar la empresa a las nuevas circunstancias económicas ( $88^{\prime} 9 \%$ ).

6.6. Organizando la realidad pata darle un sentido: tipologías de empresas andaluzas

Respondiendo al Objetivo 2 de nuestro estudio, las pruebas realizadas han posibilitado la agrupación de todas las empresas analizadas, así como la obtención de un perfil medio de las mismas. Nos encontramos con empresas donde la gestión llevada a cabo es lo más valorado.

Comienza a vislumbrarse cierto protagonismo de la formación y la innovación comienza a despuntar. El cambio cultural todavía no se concibe como una realidad presente en nuestras empresas, donde muchos aspectos todavía son abordados desde una perspectiva tradicional, sobre todo los relacionados con la estructura jerarquizada y con la concepción de que mayor productividad del trabajador equivale a mayor presencialidad de tiempo en la empresa.

La crisis económica parece que no ha tenido graves repercusiones en las empresas analizadas y en los casos que sí lo ha hecho ha sido combatida normalmente con respuestas que han provocado mejoras en las empresas (innovaciones, más formación....). 


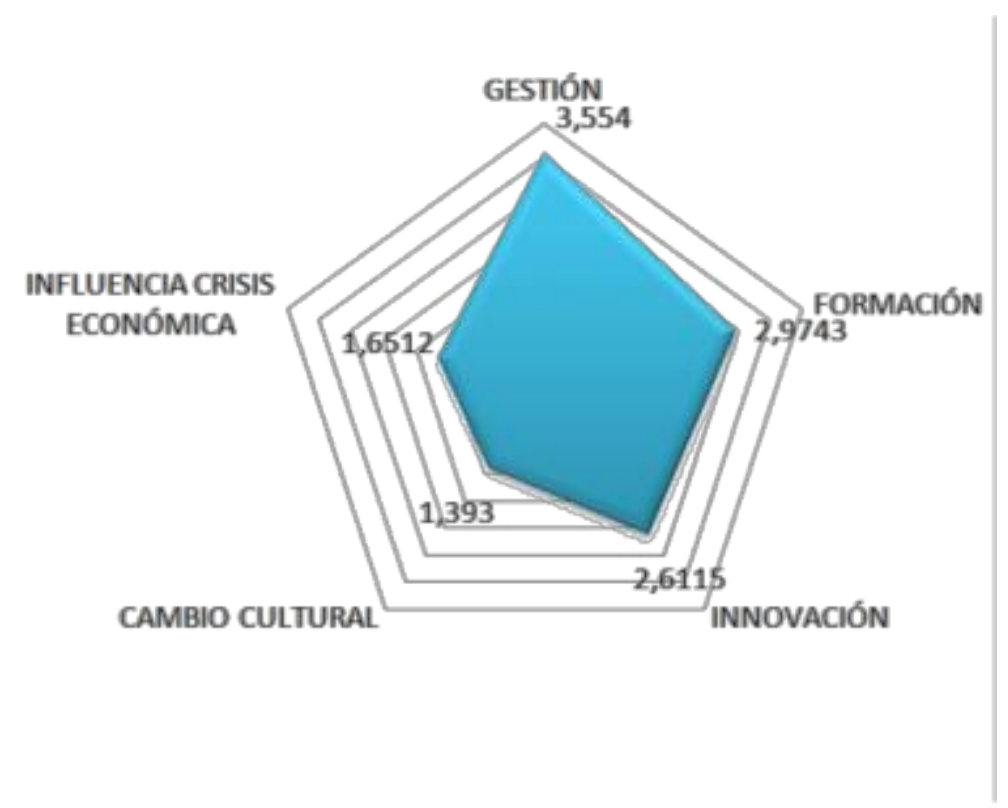

Figura 1. Gráfico de representación de la distribución del perfil medio de empresas en función de la media de los cinco componentes estudiados: Gestión, formación, innovación, cambio cultural e influencia de la crisis económica.

Aplicando la prueba del análisis de conglomerados (Figura 2) se refleja que existen tres tipos de agrupaciones de empresas:

a) Las empresas del conglomerado 1 son empresas con una buena valoración de la gestión (3’56) y una aceptable consideración de la formación (2’98). La innovación todavía no es considerada importante en su práctica diaria (1’49) y los factores de cambio cultural (0’54) y de crisis económica (0’74) parecen no haberle alcanzado, pasando prácticamente desapercibidos.

b) Las empresas del conglomerado 2 son empresas donde la innovación (0’08) y el cambio cultural $\left(0^{\prime} 00\right)$ son prácticamente imperceptibles. Poco afectadas por la crisis (0’26), no tienen en la formación (0’38) uno de sus pilares fundamentales, y perciben que la gestión (1’81) aún tiene muchos elementos que mejorar.

c) Las empresas del conglomerado 3 son empresas con una alta valoración de la gestión (4’07), y con una adecuada puesta en práctica de la formación (3’74) y la innovación (3’80) en su quehacer diario, en las que los factores de la crisis económica (2’42) no han afectado demasiado pero pueden detectarse algunas influencias de la misma y en las que a pesar de que se va percibiendo el cambio cultural, aún queda bastante por trabajar en él (2'14). 


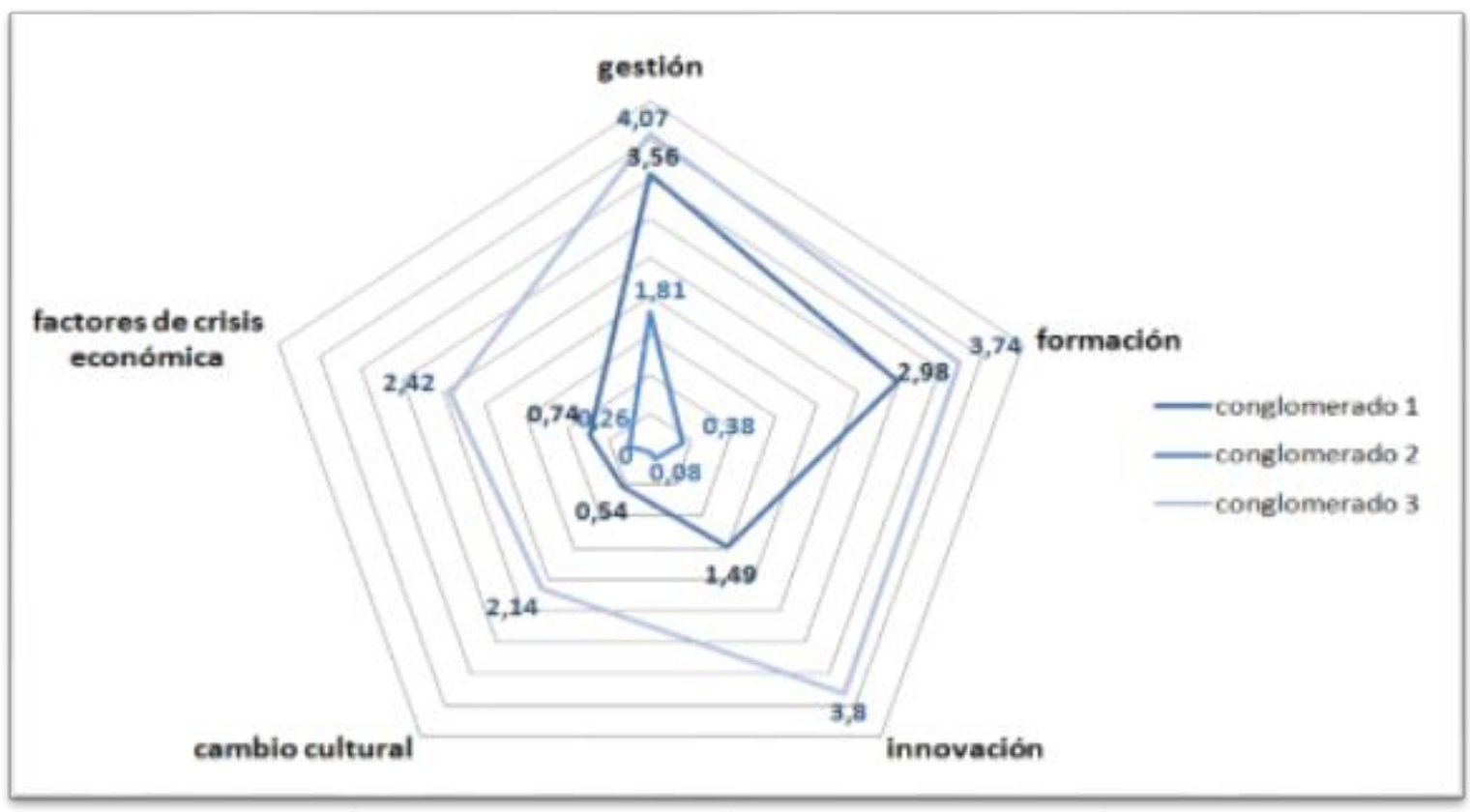

Figura 2. Prueba del análisis de conglomerados

En nuestro estudio podemos decir que las variables innovación y formación son las que ejerce mayor influencia en la formación de los conglomerados, mientras que la variable factores de la crisis es la que menos influye. Los resultados obtenidos, mostrados en la figura 3, indican el número de casos perteneciente a cada conglomerado. Así en el conglomerado 1 tenemos 29 casos, en el conglomerado 2 encontramos 22 casos y, finalmente, en el conglomerado 3 tenemos 74 casos.

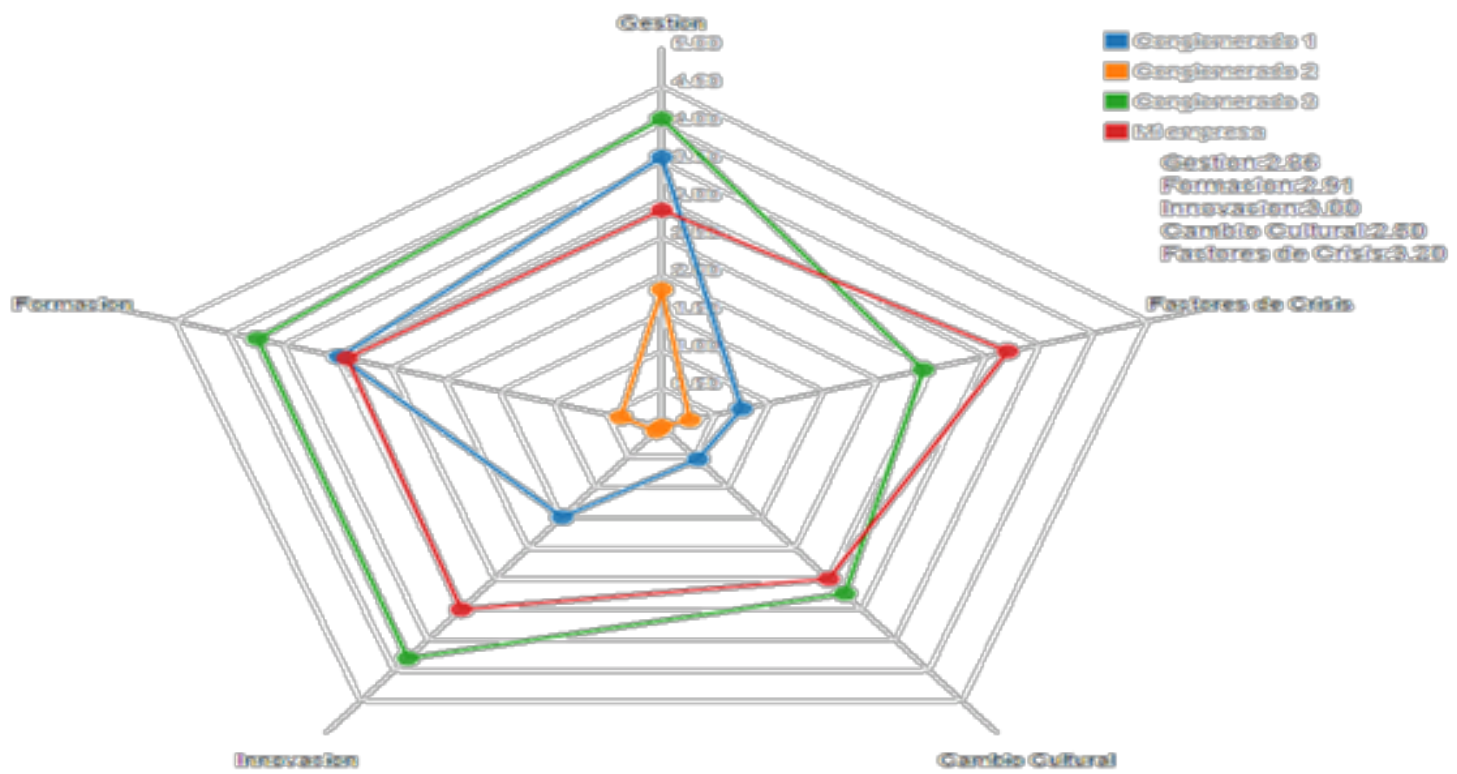

Figura 3. Prueba del análisis de conglomerados por variables 
Para concluir la exposición de resultados de nuestro estudio, hemos considerado de interés plantearnos como problema de análisis probar si existen diferencias significativas en los principales conceptos estudiados en las organizaciones en función de diferentes rasgos de la muestra: Sexo, edad y cluster o conglomerado de pertenencia. Para ello hemos aplicado a nuestros datos la prueba U de Mann-Whitney. Podemos afirmar que en función del sexo no hay diferencias. Por otra parte, también hemos aplicado la prueba de Kruskal-Wallis, que es una extensión de la de U de MannWhitney cuando existen más de dos muestras. Como resultado de la misma, podemos afirmar que en función de la edad se confirman las h0 ( $\mathrm{p}>0.05)$, y no existen diferencias. Dado que las características personales de las personas que han contestado el cuestionario no son influyentes en sus percepciones sobre los diferentes aspectos abordados en el estudio, hemos considerado interesante realizar el análisis en función del cluster de pertenencia de cada empresa. En función del cluster de pertenencia se confirman las h0 ( $\mathrm{p}<0.05)$, y sí existen diferencias.

Confirmadas nuestras hipótesis, y obteniendo como resultados que las empresas tienen distinta percepción de los factores estudiados en función del cluster al que pertenecen, hemos considerado de interés realizar otro nueva prueba estadística que nos ayude a verificar la validez de esta clasificación de las empresas y nos permita una representación gráfica del resultado que nos aporte una visión global de la agrupación de empresas en nuestra comunidad.

Vemos que se ha clasificado correctamente el 91 '2\%. El alto porcentaje (91'2\%) de las empresas que han sido clasificadas correctamente por medio de las funciones discriminantes canónicas, demuestra que éstas poseen un alto poder discriminante y pueden ser utilizadas para futuras pruebas de clasificación de empresas nuevas.

\subsection{Construcción de nuestra propia herramienta}

Nuestro Objetivo 3 consistía en la construcción de nuestra herramienta autodiagnóstica para las empresas que les permitiese posicionarse en un gráfico y comprobar su situación en función de los tipos de empresas resultantes del estudio, para a partir de ahí, orientarse hacia un itinerario formativo determinado.

Vemos representada una imagen de dicho cuestionario online (Figura 4), diseñado para el envío telemático a las empresas y que permita su rápida y cómoda cumplimentación y les permita obtener de la misma forma la representación gráfica de su empresa en relación con los tres tipos principales, identificados en el estudio:

\section{FORMULARIO PARA LA DETECCION DEL GRUPO DE PERTENENCIA DE SU EMPRESA Y LA CONSECUENTE DERIVACION A UN PLAN DE FORMACION A SU MEDIDA}

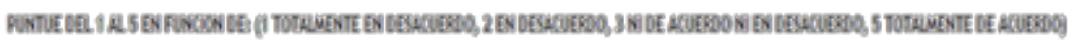

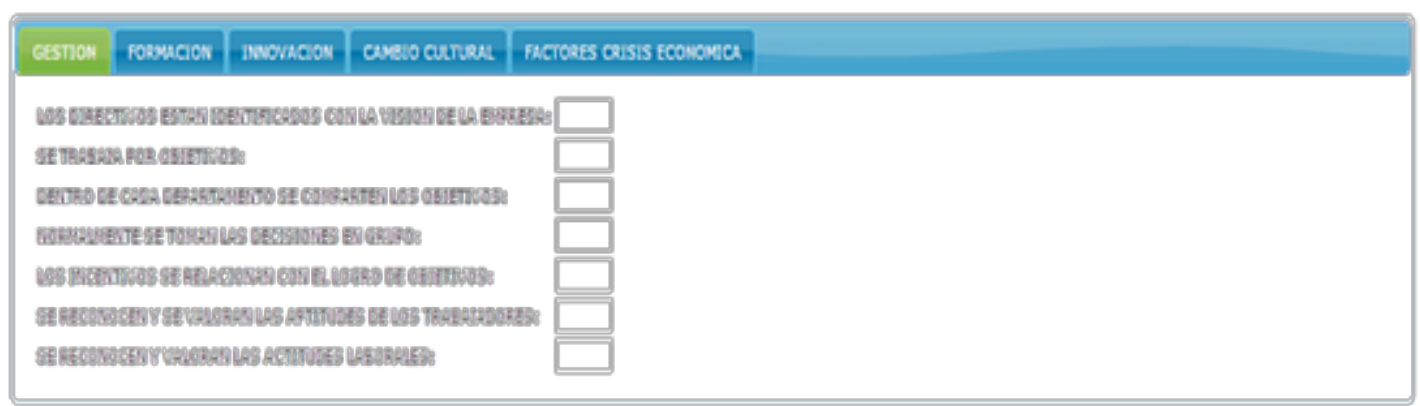

Covar

Figura 4. Formulario para la detección del grupo de pertenencia

Revista Fuentes

2016, 18 (1), 15-32 


\section{Conclusiones}

Tras los resultados expuestos nuestras conclusiones pueden resumirse así:

a) Una formación empresarial entendida según este esquema, coloca en una posición central al trabajador como objeto y objetivo de la misma, pero sin olvidar en el contexto en el que se haya inserto, y los resultados que se esperan obtener, que van más allá de la mera adquisición de conceptos o conocimientos, atendiendo tanto al desarrollo personal como empresarial y se define como aprendizaje organizacional.

b) Plantear un plan de formación no es algo puntual sino que debe realizarse dentro del plan organizacional de toda la empresa, entendiendo así la formación como un eje estratégico fundamental de la empresa, capaz de propiciar grandes mejoras, promover profundos cambios y generar enormes beneficios.

Uno de los puntos de partida de nuestro estudio, fue descubrir qué ha supuesto la incorporación del ordenador e Internet a las empresas andaluzas. Como podemos comprobar en el capítulo de resultados de la investigación, y como se observa en el mapa arriba representado, la introducción de estos nuevos componentes que llevan asociado en sí mismos la innovación y el cambio, han propiciado una pequeña revolución a muchos niveles:

- La información y la comunicación se han visto positivamente afectadas.

- La productividad ha mejorado en muchos sentidos.

- El trabajador puede beneficiarse de cierta flexibilidad laboral.

- Se refuerza el concepto de confianza en el trabajador.

- La formación continua produce un reciclaje profesional que beneficia enormemente a la empresa y a sus trabajadores.

Pero en nuestro estudio no sólo interesaba conocer qué ha supuesto la incorporación del ordenador e Internet a las empresas andaluzas sino además identificar los componentes de la cultura de las empresas: innovación, gestión de Recursos Humanos, clima laboral, formación, TIC, responsabilidad social corporativa; considerados elementos claves de las empresas que propician o pueden llegar a propiciar el cambio cultural.

Tras nuestro análisis podemos concluir que los principales elementos que están posibilitando el cambio cultural en las empresas andaluzas son:

a) La gestión

b) La formación

c) Y el uso de Internet y las TIC, que nos encaminaba a analizar si existe un cambio cultural en la empresa propiciado por estas tecnologías.

En torno a estos elementos, hay opiniones dispares, pero todas ellas apuntan hacia el cambio cultural de una u otra forma. Gracias a ellas, se percibe el cambio en aspectos tales como:

- Flexibilidad

- Responsabilidad Social Corporativa

- Estamos viendo pues, que todo esto señala hacia unas nuevas cualidades en la dirección

- La comunicación adquiere un peso importante en la organización

- Se propicia un desarrollo tanto a nivel empresarial como personal:

Una formación entendida de esta forma es también considerada un recurso económico en un doble sentido. Por una parte, necesita de inversión, sobre todo en aspectos tales como la 
investigación y el desarrollo, pero a su vez, produce resultados, también algunos beneficios económicos, por lo que se hace muy necesaria una permanente medición de la transferencia o del impacto de la formación que se realiza.

Con estas aportaciones obtenidas del análisis de las respuestas de nuestras empresas podemos decir que en gran medida, aunque a veces de forma inconsciente, los trabajadores apuestan por nuevas políticas de Recursos Humanos dentro de las empresas que se asienten en modelos de gestión basados en el aprendizaje organizativo y la gestión del conocimiento.

¿Y cuál es nuestro papel dentro de este entramado? ¿Qué puede aportar un pedagogo? Pues bien, personalmente considero que la clave del cambio está en las personas que forman la empresa. $\mathrm{Si}$ las personas que constituyen la empresa, perciben la necesidad del cambio y van intentando poco a poco ir transformando aquello que es necesario, van innovando su cultura, su forma de gestionar, su forma de organizarse, hacia formas más concienciadas, más pensadas para todos, con más responsabilidad social y personal, la empresa será otra totalmente nueva aunque siga produciendo y comercializando el mismo producto que iniciaron sus abuelos. Aquí pienso que está nuestro papel. Dentro del núcleo de las personas, dentro de ese área de Recursos Humanos, un área importante porque gestiona el activo más importante de las empresas, a las personas; y además porque de ella partirán los elementos más importantes para ese aclamado cambio: la formación, la motivación laboral, la responsabilidad social corporativa, el salario, la negociación...

\subsection{Propuesta}

Finalmente, nuestra propuesta y modelo de actuación, es incitar a todas las empresas a diseñar y desarrollar su plan de formacióan anual. Proponer un plan de formación genérico para todas las empresas de Andalucía no es una propuesta realista, ya que cada empresa cuenta con sus peculiaridades, sus necesidades y sus características que la hacen tan única, que la mejor formación es aquella que se hace a medida para ella.

Partiendo del análisis de necesidades de las personas y de la organización deben marcarse los objetivos. Nunca se pueden marcar unos objetivos formativos sin tener en cuenta las necesidades, porque de ese modo, difícilmente serán realistas y en la mayoría de los casos, perd erán el interés de las personas que deberán formarse, puesto que no partirán de una necesidad y eso les desmotivará. Posteriormente, es necesario adaptar las estrategias que se llevarán a cabo. Adaptarlas a los contenidos, a las personas y sus necesidades, al espacio, al tiempo. Estrategias funcionales que motiven y faciliten la consecución de los objetivos y la asimilación de los contenidos. Esto nos lleva a realizar una formación a medida. No pretendamos desarrollar cursos estándar, válidos para todos.

Con todos estos condicionantes previos resueltos podemos producir los contenidos. Unos contenidos que den respuesta a todo lo anterior. En este punto nos encontramos que ya tenemos "el curso", pasemos a la puesta en práctica, la realización de la formación. Con unos formadores, expertos tanto en conocimientos técnicos como en didácticos. Poseer un amplio conocimiento de la materia pero no saber transmitirla no sirve de nada. Tan importante como el desarrollo de los contenidos, es la elección del formador que los transmita y ayude a su asimilación.

En muchos casos, aquí se pone el punto y final a la formación. Un curso finaliza cuando concluye la última sesión formativa, pero no es así. Si cerramos la formación así, sin más, nos falta un apartado muy importante del proceso formativo y de un buen plan de formación: el seguimiento y la evaluación. La retroalimentación. Es necesario conocer si la formación ha funcionado, si ha cumplido los objetivos y cubierto las expectativas. Si ha dado una respuesta a las necesidades de partida, provocando los cambios y mejoras buscados.

Este seguimiento y evaluación son los que nos darán las pautas para continuar con la formación. 


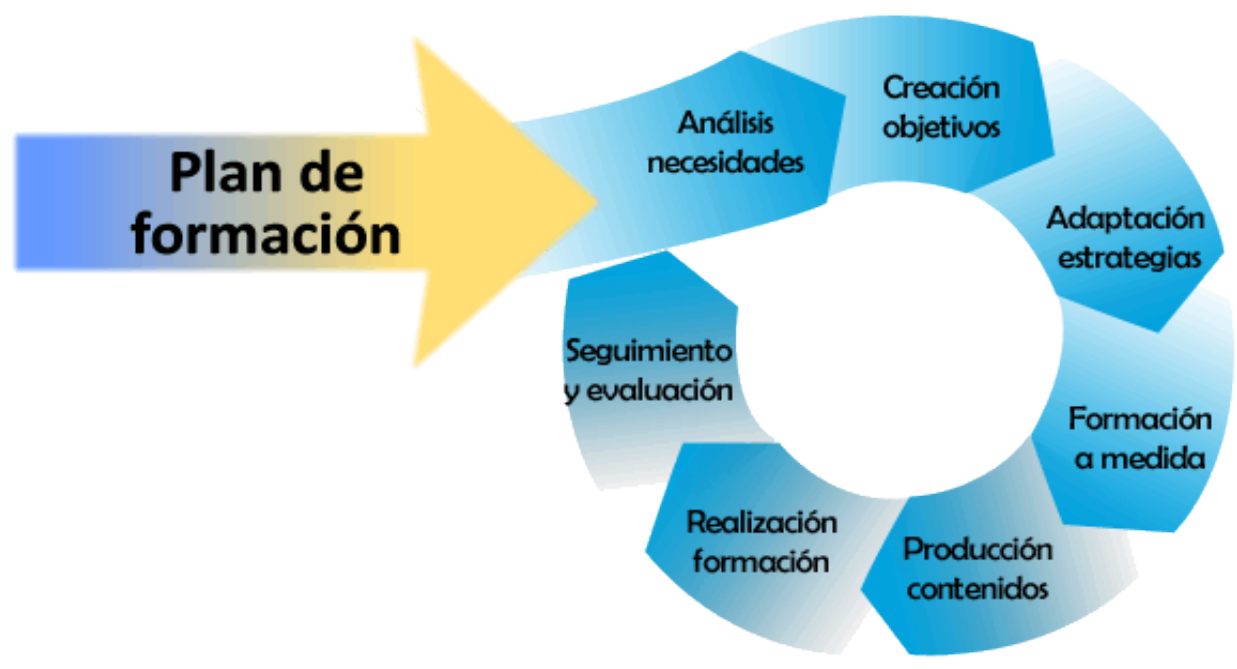

Figura 5. Diagrama plan de formación

\subsection{Aportación final}

Para terminar como aportación final decir que casi con total seguridad me atrevo a afirmar que Educación, Innovación y Estimulación Empresarial serán las claves y retos del futuro.

\section{Referencias bibliográficas}

Argandoña, A. (2010). La dimensión ética de la crisis financiera. En A. Costas Comesaña (coord.), La crisis de 2008. De la economía a la política y más allá (pp. 183-198). El Ejido (Almería): Cajamar. Recuperado

de: http://www.fundacioncajamar.com/files/publicaciones/capitulos/13.pdf

Benito-Hernández, S., Platero-Jaime, M., \& Rodríguez-Duarte, A. (2012). Factores determinantes de la innovación en las microempresas españolas: La importancia de los factores internos. Universia Business Review, 33, 104-121.

Bravo Ibarra, E. R., \& Herrera, L. (2009). Capacidad de innovación y configuración de recursos organizativos. Intangible Capital, 5 (3), 301-320.

Bravo Ibarra, E.; Herrera, L., \& Del Valle Granada, D. (2009). Un modelo de referencia de la capacidad de innovación: Un estudio de casos exploratorio en el sector audiovisual español. En XIII SeminarioLatino-Iberoamericano de Gestión Tecnológica (ALTEC 2009). Recuperado de: https://upcommons.upc.edu/bitstream/handle/2117/6414/E-Bravo.pdf

Bravo, E., \& Herrera, L. (2009). Generación de capacidades dinámicas mediante la innovación organizacional: Un múltiple estudio de casos exploratorio. En III Congreso de Ingeniería de Organización (pp. 195-205). Recuperado de: http://adingor.es/congresos/web/uploads/cio/cio2009/195-205.pdf.

Castillo, J.J. (2009). Las fábricas de software en España: organización y división del trabajo. El trabajo fluido en la sociedad de la información. Trabajo y Sociedad. Indagaciones sobre el trabajo, la cultura y las prácticas politicas en sociedades segmentadas, 12, 1-39.

De Pablos Pons, J. (2010). Universidad y Sociedad del Conocimiento. Las competencias informacionales y digitales. En M. Área Moreira (coord.), Competencias informacionales y 
digitales en educación superior. Revista de Universidad y Sociedad del Conocimiento (pp. 6-16). Barcelona: UOC.

Felber, C. (2012). La economía del bien común. Barcelona: Deusto.

Fernández, P., Aguirreamalloa, J., \& Corres, L. (2011). Rentabilidad y creación de valor de 125 empresas españolas en 2010. IESE. Universidad de Navarra.

Galbraith, J.K. (2013). El crash de 1929. Barcelona: Ariel.

Judt, T. (2010). Algo va mal. Madrid: Taurus.

Krugman, P. (2009). El retorno a la economía de la depresión y la crisis actual. Barcelona: Crítica.

Martín Castro, M. B. (2010). Reformas en el estado de bienestar mediterráneo: especial referencia al caso español en la época de crisis. Revista de la Facultad de Ciencias Sociales y Jurídicas de Elche, $8,174-201$.

Ministerio de Industria, Energía y Turismo (2014). Estadística Pyme. Evolución e indicadores. N ${ }^{\circ}$ 12. Marzo 2014. Recuperado de: www.ipyme.org

Niño Becerra, S. (2009). El crash del 2010. Toda la verdad sobre la crisis. Barcelona: Libros del Lince.

Pérez-Ruiz, A., \& Rodríguez-Del Bosque, I. (2012). La imagen de responsabilidad social corporativa en un contexto de crisis económica: El caso del sector financiero en España. Universia Business Review, 33, 14-29. Recuperado de: http://ubr.universia.net/pdfs web/3301.pdf

Piketty, T. (2014). El Capital en el Siglo XXI. Madrid: Fondo de Cultura Economica de España.

Prieto, G., \& Delgado, A. R. (2010). Fiabilidad y Validez. Papeles del Psicólogo, 31(1), 67-74. Recuperado de: http://www.cop.es/papeles

Roubini, N. Y Mihm, S. (2010). Cómo salimos de ésta. Hoja de ruta del futuro de la economía, por el experto que predijo la crisis mundial. Barcelona: Destino.

Stiglitz, J. E. (2010). Caída libre. El libre mercado y el hundimiento de la economía mundial. Madrid: Taurus.

Torrado-Fonseca, M., \& Berlanga-Silvente, V. (2013). Análisis Discriminante mediante SPSS. REIRE, Revista d'Innovació $i$ Recerca en Educació, 6(2), 150-166. Recuperado de: http://www.ub.edu/ice/ reire.htm

Van den Bergh, J. (2011). Ideas para que España salga de la crisis: un punto de vista externo. Revista de Economía Crítica, 12,171 - 176.

\section{Para citar este artículo}

Romera Hiniesta, F. (2016). Estudio sobre las organizaciones inteligentes en Andalucía. Revista Fuentes, 18(1), 15-32. [Fecha de consulta: dd/mm/aa]. doi: doi: http://dx.doi.org/10.12795/revistafuentes.2016.18.1.01 